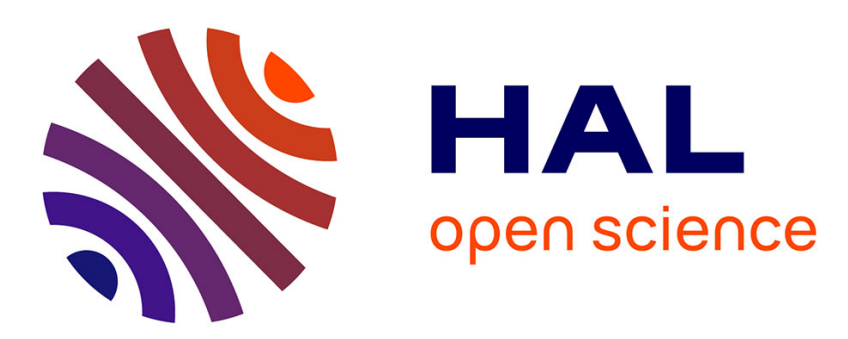

\title{
Carrier escape from ground state and non-zero resonance frequency at low bias powers for semiconductor quantum-dot lasers
}

Cheng Wang, Frederic Grillot, Jacky Even

\section{- To cite this version:}

Cheng Wang, Frederic Grillot, Jacky Even. Carrier escape from ground state and non-zero resonance frequency at low bias powers for semiconductor quantum-dot lasers. SPIE Photonics Europe, Apr 2012, brussels, Belgium. hal-00805233

\section{HAL Id: hal-00805233 \\ https://hal.science/hal-00805233}

Submitted on 28 Mar 2013

HAL is a multi-disciplinary open access archive for the deposit and dissemination of scientific research documents, whether they are published or not. The documents may come from teaching and research institutions in France or abroad, or from public or private research centers.
L'archive ouverte pluridisciplinaire HAL, est destinée au dépôt et à la diffusion de documents scientifiques de niveau recherche, publiés ou non, émanant des établissements d'enseignement et de recherche français ou étrangers, des laboratoires publics ou privés. 


\title{
Carrier escape from ground state and non-zero resonance frequency at low bias powers for semiconductor quantum-dot lasers
}

\author{
C. Wang ${ }^{a^{*}}$, F. Grillot ${ }^{\mathrm{a}, \mathrm{b}}$ and J. Even ${ }^{\mathrm{a}}$ \\ ${ }^{a}$ Université Européenne de Bretagne, INSA, CNRS FOTON, 20 avenue des buttes de Coesmes, \\ 35708 Rennes Cedex 7, France \\ ${ }^{\mathrm{b}}$ Telecom Paristech, Ecole Nationale Supérieure des Télécommunications, CNRS LTCI, 46 rue \\ Barrault, 75013 Paris, France
}

\begin{abstract}
The three-dimensional confinement of electrons and holes in the semiconductor quantum dot (QD) structure profoundly changes its density of states compared to the bulk semiconductor or the thin-film quantum well (QW) structure. The aim of this paper is to theoretically investigate the microwave properties of $\operatorname{InAs} / \operatorname{InP}(311 \mathrm{~B})$ QD lasers. A new expression of the modulation transfer function is derived for the analysis of QD laser modulation properties based on a set of four rate equations. Analytical calculations point out that carrier escape from ground state (GS) to excited state (ES) induces a non-zero resonance frequency at low bias powers. Calculations also show that the carrier escape leads to a larger damping factor offset as compared to conventional QW lasers. These results are of prime importance for a better understanding of the carrier dynamics in QD lasers as well as for further optimization of low cost sources for optical telecommunications.
\end{abstract}

Keywords: laser, quantum dot, semiconductor, modulation response

\section{INTRODUCTION}

Self-organised quantum dot (QD) lasers are promising laser sources for optical fibre communications because of their attractive characteristics, such as low threshold current density [1], high differential gain [2], high bandwidth [3,4] and low chirp [5,6]. QD lasers are expected to be alternatives of the quantum well (QW) counterparts for optical telecommunications. However, the QD lasers have not realized the expected dynamic properties, and the maximum modulation bandwidth remains limited to $10-12 \mathrm{GHz}[7,8]$ at wavelengths in 1300-1500 nm bands, much below the best reported values for QW lasers. Therefore, it is necessary to clarify the origins of limited modulation bandwidth of QD lasers, and then to find strategies for improving the modulation bandwidth to meet the network requirement. The modulation bandwidth is strongly dependent on resonance frequency and damping factor. The resonance frequency has been demonstrated to be limited by the maximum modal gain and nonlinear gain effects [9]. In particular, QD lasers are commonly observed to exhibit a stronger damping compared to QW lasers [10,11,12], which limits their maximum modulation bandwidth. The underlying physical mechanisms are still under investigation, carrier capture dynamics $[13,14,15]$, Coulomb interaction [12] and gain compression [11] are proposed to the origins. The modulation transfer function derived from the two standard QW rate equations [9] is an efficient tool for the analysis of QW laser modulation characteristics, however, it does not work well on QD lasers because of the more complicated carrier dynamics. One theoretical approach to study QD laser dynamics is to simulate the modulation properties numerically based on a set of rate equations [12]. These equations, however, are complicated for a direct understanding of the QD laser modulation response, so a simple model describing the essential QD dynamical properties is desired. Sugawara et. al proposed an empirical expression to fit the experimental modulation response [16]. Foire et. al developed an indirect approach to recast the set of complex QD rate equations into the standard QW rate equations [8]. This paper aims to theoretically investigate the microwave properties of InAs/InP(311B) QD lasers [17]. Based on a set of four rate differential equations [18], we developed a new analytical modulation transfer function, which includes the impacts of wetting layer (WL) and excited state (ES). Definitions of relaxation resonance frequency and damping factor are improved as well. The analytical analysis shows that carrier escape from GS to ES is responsible for the commonly observed strong damping of QD lasers, and leads to a non-zero resonance frequency at low bias powers. These results are significant for a better understanding of carrier dynamics of QD lasers and point to possible routes for the improvement of QD laser dynamic properties.

* cheng.wang@insa-rennes.fr; phone +33 223238464; 


\section{STEAY-STATE PROPERTIES AND TURN-ON DELAY PROPERTIES}

\subsection{Numerical model description}

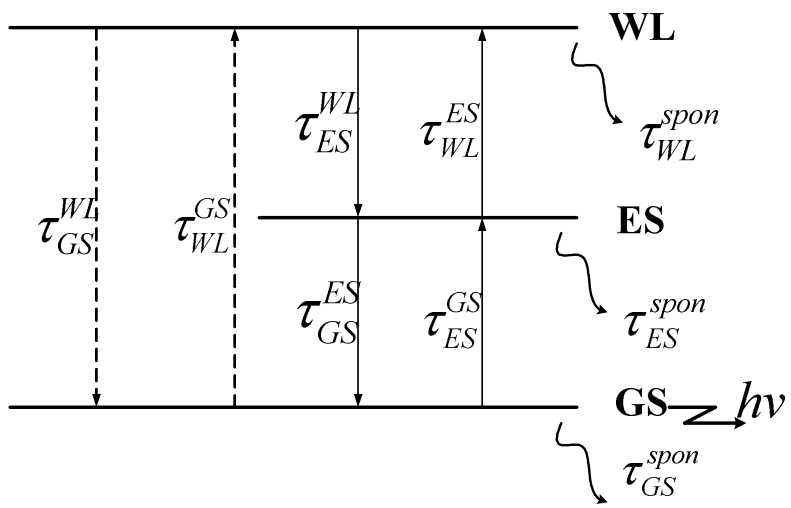

Figure 1. Illustration of carrier dynamics model including a direct relaxation channel (dash lines)

Figure 1 shows the schematic of the carrier dynamics for the InAs/InP(311B) QD laser [18]. It is assumed that there is only one QD ensemble, and the QDs are always neutral, electrons and holes being treated as electron-hole (eh) pairs. There are only one two-fold degenerated GS and one lowest four-fold degenerated ES in the QDs. GS laser emission occurs when the threshold is reached, and the stimulated emission from the ES is not taken into account in the model. Carriers are supposed to be injected directly from the contacts into the WL levels, so the barrier dynamics are not considered. Then, carriers are either captured into ES within time $\tau_{E S}^{W L}$ or directly into GS within time $\tau_{G S}^{W L}$. Once in the ES level, carriers can also relax to GS within time $\tau_{G S}^{E S}$. On the other hand, the eh-pairs recombine spontaneously or escape from GS and ES within times $\tau_{E S}^{G S}, \tau_{W L}^{G S}$ and $\tau_{W L}^{E S}$, and these times are decided by the Fermi distribution for the quasi-thermal equilibrium without external excitation [19]. Following the sketch of figure 1, the four rate equations on carrier and photon number are as follows:

$$
\begin{aligned}
\frac{d N_{W L}}{d t} & =\frac{I}{q}+\frac{N_{E S}}{\tau_{W L}^{E S}}-\frac{N_{W L}}{\tau_{E S}^{W L}} f_{E S}-\frac{N_{W L}}{\tau_{G S}^{W L}} f_{G S}-\frac{N_{W L}}{\tau_{W L}^{s p o n}}+\frac{N_{G S}}{\tau_{W L}^{G S}} \\
\frac{d N_{E S}}{d t} & =\frac{N_{W L}}{\tau_{E S}^{W L}} f_{E S}+\frac{N_{G S}}{\tau_{E S}^{G S}} f_{E S}-\frac{N_{E S}}{\tau_{W L}^{E S}}-\frac{N_{E S}}{\tau_{G S}^{E S}} f_{G S}-\frac{N_{E S}}{\tau_{E S}^{s p o n}} \\
\frac{d N_{G S}}{d t} & =\frac{N_{W L}}{\tau_{G S}^{W L}} f_{G S}+\frac{N_{E S}}{\tau_{G S}^{E S}} f_{G S}-\frac{N_{G S}}{\tau_{E S}^{G S}} f_{E S}-\frac{N_{G S}}{\tau_{G S}^{s p o n}}-g_{G S} \nu_{g} S_{G S}-\frac{N_{G S}}{\tau_{W L}^{G S}} \\
\frac{d S_{G S}}{d t} & =\Gamma_{p} g_{G S} v_{g} S_{G S}-\frac{S_{G S}}{\tau_{P}}+\Gamma_{p} \beta_{S P} \frac{N_{G S}}{\tau_{G S}^{s p o n}}
\end{aligned}
$$

where $N_{W L, E S, G S}$ are carrier numbers in WL, ES, GS and $S_{G S}$ is photon number. $\tau_{W L, E S, G S}^{\text {spon }}$ are spontaneous emission times, $\beta_{S P}$ is spontaneous emission factor and $\Gamma_{p}$ is optical confinement factor. $v_{g}$ is the group velocity and $\tau_{p}$ is photon lifetime. The GS gain is described as follows:

$$
g_{G S}=a_{G S} N_{B}\left(\frac{N_{G S}}{N_{B}}-1\right)
$$

where $a_{G S}$ is the differential gain and $N_{B}$ is the total QD number. $f_{G S, E S}$ are the probabilities to find an empty carrier state in GS and ES:

$$
f_{G S}=1-\frac{N_{G S}}{2 N_{B}} ; \quad f_{E S}=1-\frac{N_{E S}}{4 N_{B}}
$$

Since the carrier escape from GS to WL has been demonstrated to have little effects on laser properties [18], the $N_{G S} / \tau_{W L}^{G S}$ term in Eq. (1) and Eq. (3) can be neglected. 


\subsection{Steady-state properties and turn-on delay properties}

Steady-state properties as well as turn-on delay properties are studied by solving the four rate equation model numerically. The capture time $\tau_{E S}^{W L}$ and relaxation time $\tau_{G S}^{E S}$ are fixed from the time resolved photoluminescence experiment [20]. The direct carrier capture time from WL to GS $\tau_{G S}^{W L}$ is observed to be larger than the capture time $\tau_{E S}^{W L}$ in low excitation regime [21], while in strong excitation regime $\tau_{G S}^{W L}$ becomes as short as $\tau_{E S}^{W L}$, i. e. $\tau_{G S}^{W L} \approx \tau_{E S}^{W L}$ [20]. In our calculation, we take $\tau_{G S}^{W L}=1.5 \tau_{E S}^{W L}$ and the differential gain $\mathrm{a}_{\mathrm{GS}}=0.5 \times 10^{-14} \mathrm{~cm}^{2}$. Other simulation parameters are summarized in table 1 .

Table 1. The QD material parameters and the laser parameters

\begin{tabular}{|ll|ll|}
\hline \multicolumn{2}{|c|}{ QD material parameters } & \multicolumn{2}{c|}{ Laser parameters } \\
\hline WL energy: & $\mathrm{E}_{\mathrm{WL}}=0.97 \mathrm{eV}$ & Active region length: & $\mathrm{L}=0.11 \mathrm{~cm}$ \\
\hline ES energy: & $\mathrm{E}_{\mathrm{ES}}=0.87 \mathrm{eV}$ & Active region width: & $\mathrm{W}=3 \times 10^{-4} \mathrm{~cm}$ \\
\hline GS energy: & $\mathrm{E}_{\mathrm{GS}}=0.82 \mathrm{eV}$ & Number of QD layers: & $\mathrm{N}=5$ \\
\hline Capture time from WL to ES: & $\tau_{E S}^{W L}=25.1 \mathrm{ps}$ & QD density: & $\mathrm{N}_{\mathrm{D}}=5 \times 10^{10} \mathrm{~cm}^{-2}$ \\
\hline Relaxation time from ES to GS: & $\tau_{G S}^{E S}=11.6 \mathrm{ps}$ & Optical confinement factor: & $\Gamma_{p}=0.06$ \\
\hline Spontaneous time of WL and ES: & $\tau_{W L}^{\text {spon }}=\tau_{E S}^{\text {spon }}=500 \mathrm{ps}$ & Spontaneous emission factor: & $\beta_{S P}=1 \times 10^{-4}$ \\
\hline Spontaneous time of GS: & $\tau_{G S}^{\text {spon }}=1200 \mathrm{ps}$ & Internal modal loss: & $\alpha_{i}=6 \mathrm{~cm}^{-1}$ \\
\hline Refractive index: & $\mathrm{n}_{\mathrm{r}}=3.27$ & Mirror reflectivity: & $\mathrm{R}_{1}=\mathrm{R}_{2}=0.3$ \\
\hline
\end{tabular}

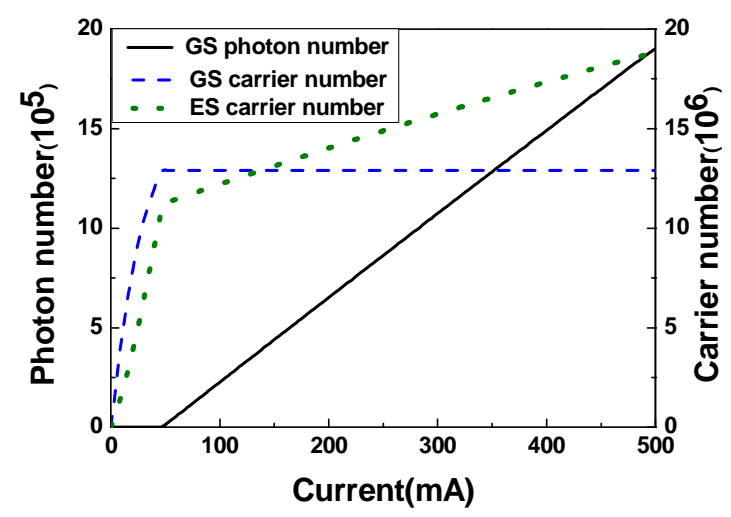

Figure 2. Calculated photon number and carrier number as a function of injected current.

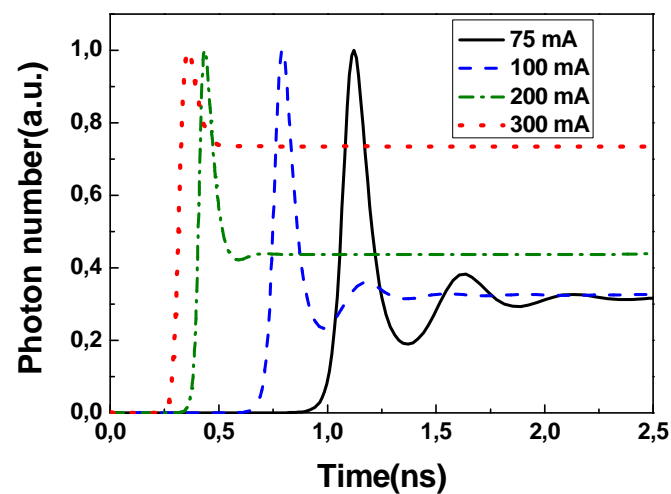

Figure 3. Turn-on delay of the GS lasing at various current injection levels. 
Steady-state properties of the InAs/InP (311B) QD laser are shown in figure 2. With the increased current injection, both the carrier populations in GS and ES are enhanced. Then, above an injected current of about $48 \mathrm{~mA}$, the GS population gets clamped which leads to the occurrence of the GS lasing emission, while the ES population continues increase with a decreased slope efficiency. Figure 3 presents the transient responses of GS lasing at several current injections. With the increase of injected current, the delay time becomes shorter which indicates a decreased carrier lifetime [22]. Both the oscillation frequency and the damping factor increase with the increasing current. The oscillation frequency at $75 \mathrm{~mA}$, $100 \mathrm{~mA}$ and $200 \mathrm{~mA}$ are $1.94 \mathrm{GHz}, 2.64 \mathrm{GHz}$ and $3.52 \mathrm{GHz}$, respectively. The relaxation oscillation cannot be distinguished at $300 \mathrm{~mA}$ due to the strong damping of relaxation oscillations.

\section{SMALL-SIGNAL ANALYSIS OF INTENSITY MODULATION RESPONSE}

\subsection{Analytical derivations}

Based on the set of rate equations (1-4) and considering $I, N_{W L}, N_{E S}, N_{G S}, S_{G S}$ as well as $g_{G S}$ as dynamic variables, we can derive the differential rate equations on carrier and photon density:

$$
\frac{d}{d t}\left[\begin{array}{c}
d N_{W L} \\
d N_{E S} \\
d N_{G S} \\
d S_{G S}
\end{array}\right]=\left[\begin{array}{cccc}
-\gamma_{11} & \gamma_{12} & 0 & 0 \\
\gamma_{21} & -\gamma_{22} & \gamma_{23} & 0 \\
\gamma_{31} & \gamma_{32} & -\gamma_{33} & \gamma_{34} \\
0 & 0 & \gamma_{43} & -\gamma_{44}
\end{array}\right]\left[\begin{array}{c}
d N_{W L} \\
d N_{E S} \\
d N_{G S} \\
d S_{G S}
\end{array}\right]+\frac{d I}{q V}\left[\begin{array}{l}
1 \\
0 \\
0 \\
0
\end{array}\right]
$$

where

$$
\begin{aligned}
& \gamma_{11}=\frac{f_{E S}}{\tau_{E S}^{W L}}+\frac{f_{G S}}{\tau_{G S}^{W L}}+\frac{1}{\tau_{W L}^{s p o n}} ; \quad \gamma_{12}=\frac{1}{\tau_{W L}^{E S}} ; \quad \gamma_{21}=\frac{f_{E S}}{\tau_{E S}^{W L}} \\
& \gamma_{22}=\frac{f_{G S}}{\tau_{G S}^{E S}}+\frac{1}{\tau_{W L}^{E S}}+\frac{1}{\tau_{E S}^{s p o n}} \\
& \gamma_{23}=\frac{f_{E S}}{\tau_{E S}^{G S}} ; \gamma_{31}=\frac{f_{G S}}{\tau_{G S}^{W L}} ; \gamma_{32}=\frac{f_{G S}}{\tau_{G S}^{E S}} \\
& \gamma_{33}=\frac{f_{E S}}{\tau_{E S}^{G S}}+\frac{1}{\tau_{G S}^{s p o n}}+v_{g} a_{G S} S_{G S} ; \gamma_{34}=v_{g} a_{G S}^{p} S_{G S}-v_{g} g_{G S} \\
& \gamma_{43}=\frac{\Gamma_{p} \beta_{S P}}{\tau_{G S}^{s p o n}}+\Gamma_{p} v_{g} a_{G S} S_{G S} ; \gamma_{44}=\Gamma_{p} v_{g} a_{G S}^{p} S_{G S}-\Gamma_{p} v_{g} g_{G S}+\frac{1}{\tau_{p}}
\end{aligned}
$$

where the gain variation is $d g_{G S}=a_{G S} d N_{G S}-a_{G S}^{p} d S_{G S}$, and $a_{G S}^{p}=-\partial g_{G S} / \partial S_{G S}$. Applying a small-signal analysis to the differential rate equations allows extracting a new expression of the QD laser's modulation response:

$$
H(w) \equiv \frac{R_{0}}{R_{0}+j w R_{1}-w^{2} R_{2}-j w^{3} R_{3}+w^{4}}
$$

The four parameters which characterize $H(w)$ are given by:

$$
\begin{aligned}
& R_{0}=w_{R}^{2}\left(\gamma_{11} \gamma_{22}-\gamma_{12} \gamma_{21}\right)-\gamma_{23} \gamma_{44}\left(\gamma_{31} \gamma_{12}+\gamma_{11} \gamma_{32}\right) \\
& R_{1}=w_{R}^{2}\left(\gamma_{11}+\gamma_{22}\right)+\Gamma\left(\gamma_{11} \gamma_{22}-\gamma_{12} \gamma_{21}\right)-\gamma_{23} \gamma_{32}\left(\gamma_{11}+\gamma_{44}\right)-\gamma_{31} \gamma_{12} \gamma_{23} \\
& R_{2}=w_{R}^{2}+\Gamma\left(\gamma_{11}+\gamma_{22}\right)+\left(\gamma_{11} \gamma_{22}-\gamma_{12} \gamma_{21}\right)-\gamma_{23} \gamma_{32} \\
& R_{3}=\Gamma+\left(\gamma_{11}+\gamma_{22}\right)
\end{aligned}
$$

And the improved relaxation resonance frequency $w_{R}$ and damping factor $\Gamma$ are defined as

$$
\begin{aligned}
w_{R}^{2} & =\gamma_{33} \gamma_{44}-\gamma_{34} \gamma_{43} \\
\Gamma & =\gamma_{33}+\gamma_{44}
\end{aligned}
$$

Using the set of equations (8), Eq. (11) and Eq. (12) can be re-expressed as follows: 


$$
\begin{gathered}
w_{R}^{2}=\frac{v_{g} a_{G S} S_{G S}}{\tau_{p}}+\left[\Gamma_{p} v_{g} a_{G S}^{p} S_{G S}+\frac{\Gamma_{p} \beta_{S P} N_{G S}}{\tau_{G S}^{s p o n} S_{G S}}\right]\left(\frac{f_{E S}}{\tau_{E S}^{G S}}+\frac{1-\beta_{S P}}{\tau_{G S}^{s p o n}}\right)+\frac{\beta_{S P}}{\tau_{G S}^{s p o n} \tau_{p}} \\
\Gamma=v_{g} a_{G S} S_{G S}\left[1+\frac{\Gamma_{p} a_{G S}^{p}}{a_{G S}}\right]+\frac{f_{E S}}{\tau_{E S}^{G S}}+\frac{1}{\tau_{G S}^{s p o n}}+\frac{\Gamma_{p} \beta_{S P} N_{G S}}{\tau_{G S}^{s p o n} S_{G S}}
\end{gathered}
$$

where we have used the steady-state relation $1 / \tau_{p}-\Gamma_{p} v_{g} g_{G S}=\Gamma_{p} \beta_{S P} N_{G S} / \tau_{G S}^{s p o n} S_{G S}$. It is important to note that the improved resonance frequency and damping factor differ from those for QW lasers [9]. For the QD structure, $w_{R}^{2}$ and $\Gamma$ contain an additional term $f_{E S} / \tau_{E S}^{G S}$, i. e. carrier escape from the GS to the ES. Since the first term in $w_{R}^{2}$ dominates over all the other terms, the resonance frequency can be reduced to $w_{R}^{2} \approx v_{g} S_{G S} a_{G S} / \tau_{p}$. Employing this simplified definition of $w_{R}^{2}$ and rewrite the damping factor:

where the so-called K-factor is as follows:

$$
\Gamma=K f_{R}^{2}+\frac{f_{E S}}{\tau_{E S}^{G S}}+\frac{1}{\tau_{G S}^{s p o n}}+\frac{\Gamma_{p} \beta_{S P} N_{G S}}{\tau_{G S}^{s p o n} S_{G S}}
$$

$$
K=4 \pi^{2} \tau_{p}\left(1+\frac{\Gamma a_{G S}^{p}}{a_{G S}}\right)
$$

The expression of $\mathrm{K}$-factor is found to be the same as of the conventional one for QW lasers. The term $f_{E S} / \tau_{E S}^{G S}$ in damping factor $\Gamma$ dominates in the offset and is comparable to $K f_{R}^{2}$ even at high bias powers, so the offset can not be neglected.

Based on the steady-state results in the former section, the modulation responses are calculated from Eq. (9) for various current levels and depicted in figure 4. Numerical results show that both the relaxation frequency and the damping factor increase with the pump current. At a current injection of around $135 \mathrm{~mA}$, the modulation bandwidth reaches the maximum value $\sim 5.5 \mathrm{GHz}$, which is in good agreement with the commonly measure values $[23,24,25]$.

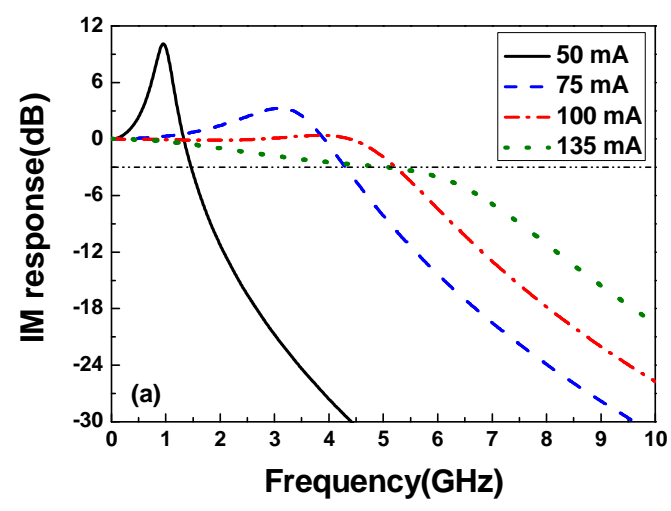

Figure 4. Intensity modulation response at several injected currents.

\subsection{Experimental results and simulations}

The studied laser device under experiment is a $\operatorname{InAs} / \operatorname{InP}(311 \mathrm{~B})$ QD laser [17], where the heterostructure is grown by MBE on a (311)B oriented InP substrate and it has as-cleaved facet. The active region consists of 5 QD layers, and the dot density is $\sim 10^{11} \mathrm{~cm}^{2}$ [26]. The length and width of the ridge wave-guide FP laser are $1.1 \mathrm{~mm}$ and $3 \times 10^{-3} \mathrm{~mm}$, respectively. Experiments show a GS lasing peak at $1.52 \mu \mathrm{m}$ at room temperature in continuous wave (cw) operation and the photon lifetime is measured to be $5.8 \mathrm{ps}$. In this section, we use the new analytical transfer function Eq. (9) to simulate the laser modulation performance. In the calculations, the differential gain $a_{G S}$ is the only fitting parameter which is adjusted to $0.25 \times 10^{-14} \mathrm{~cm}^{2}$, other parameters are set to the experimental values. 


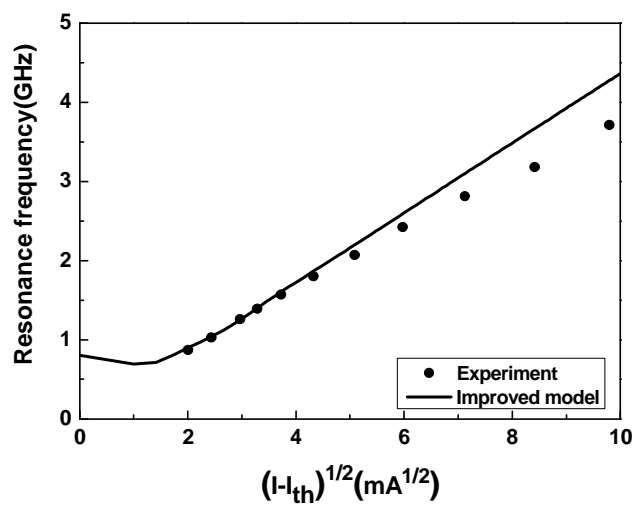

Figure 5. The resonance frequency as a function of the normalized current. Dots are the experimental results [17] and the solid line corresponds to the theoretical result.

Figure 5 shows that the resonance frequency $f_{R}$ as a function of the normalized current $\left(I-I_{t h}\right)^{1 / 2}$. Numerical results (solid line) obtained from Eq. (9) lead to a relative good agreement with the experimental results (dots). However, at large current injections, the calculated resonance frequency is higher than the experimental result, this is because the gain compression is not considered in our model. Analytical analysis points out that the carrier escape from GS to ES induces a non-zero resonance frequency around $1 \mathrm{GHz}$ at low bias powers. This resonance frequency offset is larger than the one due to spontaneous emission only in QW lasers [9].

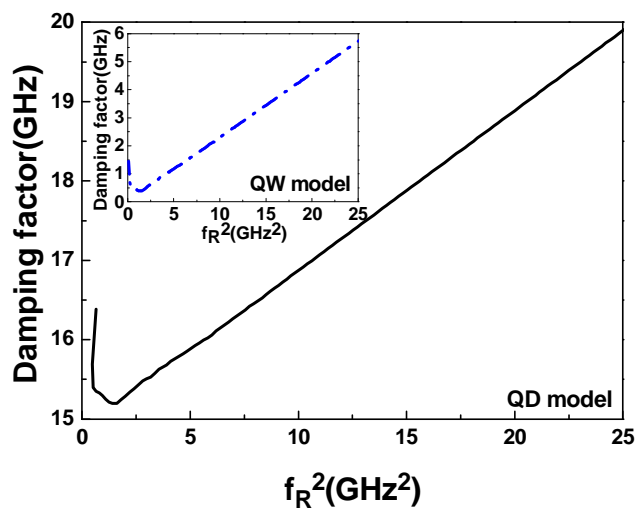

Figure 6. Comparisons of damping factor from our improved QD model and that from the QW model (inset).

Figure 6 shows the evolution of damping factor $\Gamma$ as a function of resonance frequency $f_{R}^{2}$ : the relation calculated from our improved QD model is $\Gamma=0.20 f_{R}^{2}+14.9(\mathrm{GHz})$, in comparison with the result from conventional QW model (inset): $\Gamma=0.23 f_{R}^{2}+0.066(\mathrm{GHz})$. The two $\mathrm{K}$-factors are nearly the same $\sim 0.2 \mathrm{~ns}$, which is 3 times smaller than the experimental value $0.6 \mathrm{~ns}$ [17]. Such a discrepancy can be attributed to the fact that the simulation does not take into account the gain saturation effects. Further studies will investigate the influence of $\Gamma a_{G S}^{p}$ which is comparable to the differential gain $a_{G S}$ [9]. The offset of damping factor from the QD model is much larger than that from the QW model, according to Eq. (15), this is attributed to the carrier escape from GS to ES $\left(f_{E S} / \tau_{E S}^{G S}\right)$. The large values can well indicate the commonly observed strong damping in QD lasers [10,12]. The deviation from linearity at low relaxation resonance frequency is because of the spontaneous emission term $\left(\Gamma_{p} \beta_{S P} N_{G S}\right) /\left(\tau_{G S}^{\text {spon }} S_{G S}\right)$ in the damping factor expression. This phenomenon has been observed in InGaAsP bulk lasers by $\mathrm{Su}$ et. al via a parasitic-free optical modulation technique [27]. 


\section{CONCLUSION}

A new analytical expression of the modulation response has been derived for QD lasers, which includes the impacts of wetting layer, excited states and ground state. We also improved the definitions of resonance frequency and damping factor for QD lasers. From the analysis, it is found that at low bias powers, carrier escape from GS to ES gives rise to a non-zero resonance frequency associated with a strong damping rate. These results are of first importance for a better understanding of the carrier dynamics in QD lasers for high-speed applications, and point to possible routes for the improvement of QD laser dynamics as well.

\section{REFERENCES}

[1] Liu, G. T., Stintz, A., Li, H., Malloy, K. J., and Lester, L. F. ,"Extremely low room-temperature threshold current density diode lasers using InAs dots in In Ga As quantum well," Electron. Lett. 35(14), 1163-1165 (1999).

[2] Bimberg, D., Kirstaedter, N., Ledentsov, N. N., Alferov, Z. I., Kopev, P. S., and Ustinov, V. M., "InGaAs-GaAs quantum-dot lasers," IEEE J. Sel. Topics Quantum Electron. 3(2), 196-205 (1997).

[3] Mi, Z., Bhattacharya, P., and Fathpour, S., "High-speed $1.3 \mu \mathrm{m}$ tunnel injection quantum-dot lasers," Appl. Phys. Lett. 86, 153109 (2005).

[4] Kuntz, M., Fiol, G., Lammlin, M., Schubert, C., Kovsh, A. R., Jacob, A., Umbach, A., and Bimberg, D., "10Gbit/s data modulation using 1.3 $\mu \mathrm{m}$ InGaAs quantum dot lasers," Electron. Lett. 41, 244-245 (2005).

[5] Saito, H., Nishi, K., Kamei, A., and Sugou, S., "Low chirp observed in directly modulated quantum dot lasers," IEEE Phot. Tech. Lett. 12(10), 1298-1300 (2000).

[6] Ghosh, S., Pradhan, S., and Bhattacharya, P., "Dynamic characteristics of high-speed In0.4Ga0.6As/GaAs selforganized quantum dot lasers at room temperature," Appl. Phys. Lett. 81, 3055-3057 (2002).

[7] Grillot, F., Dagens, B., Provost, J.G., Su, H., and Lester, L. F., "Gain compression and above-threshold linewidth enhancement factor in 1.3 $\mu \mathrm{m}$ InAs-GaAs quantum dot lasers," IEEE Journal of Quantum Electronics, 44(10), 946-951 (2008).

[8] Foire, A., and Markus, A., "Differential gain and gain compression in quantum-dot lasers," IEEE Journal of Quantum Electronics, 43(3), 287-294 (2007).

[9] Coldren, L. A., and Corzine, S. W., [Diode Lasers and Photonic Integrated Circuits], John Wiley \& Sons, New York, 195-204 (1995).

[10] Kuntz, M., Ledentsov, N. N., Bimberg, D., Kovsh, A. R., Ustinov, V. M., "Spectrotemporal response of $1.3 \mu \mathrm{m}$ quantum-dot lasers," Appl. Phys. Lett. 81(20), 3846-3848 (2002).

[11] O'Brien, D., Hegarty, S.P., Huyet, G., McInerney, J.G., Kettler, Laemmlin, T., Bimberg, D., Ustinov, V.M., Zhukov, A.E., Mikhrin, A.R., and Kovsh, A.R., "Feedback sensitivity of $1.3 \mu \mathrm{m}$ InAs/GaAs quantum dot laser," Electron. Lett. 39 (25), 1819-1820 (2003).

[12] Malic, E., Ahn, K.J., Bormann, M. J., Hovel, P., Scholl, E., Knorr, A., Kuntz, M., and Bimberg, D., "Theory of relaxation oscillations in semiconductor quantum dot lasers," Appl. Phys. Lett. 89, 101107 (2006)

[13] Klotzkin, D., Kamath, K., and Bhattacharya, P., "Quantum capture times at room temperature in high-speed InGa As-GaAs self-organized quantum-dot lasers," IEEE Phot. Tech. Lett. 9(10), 1301-1303 (1997)

[14] Ishida, M., Hatori, N., Akiyama, T., Otsubo, K., Nakata, Y., Ebe, H., Sugawara, M., and Arakawa, Y., "Photon lifetime dependence of modulation efficiency and $\mathrm{K}$ factor in $1.3 \mu \mathrm{m}$ self-assembled InAs/GaAs quantum-dot lasers: Impact of capture time and maximum modal gain on modulation bandwidth," Appl. Phys. Lett. 85, 4145-4147 (2004)

[15] O’Brien, D., Hegarty, S.P.,Huyet, G., and Uskov, A.V., "Sensitivity of quantum dot semiconductor lasers to optical feedback, " Opt. Lett. 29, 1072-1074 (2004).

[16] Sugawara, M. [Self-Assembled InGaAs-GaAs Quatum Dots], Academic press, San Diego, CA, (1999).

[17] Martinez, A., Merghem, K., Bouchoule, S., Moreau, G., Ramdane, A., Provost, J. G., Alexandre, F., Grillot, F., Dehaese, O., Piron, R. and Loualiche, S., "Dynamic properties of InAs/InP(311B) quantum dot Fabry-Perot lasers emitting at 1.52- $\mu \mathrm{m}$ ”, Appl. Phys. Lett., 93, 021101 (2008).

[18] Veselinov, K., Grillot, F., Cornet, C., Even, J., Bekiarski, A., Gioannini, M. and Loualiche, S., "Analysis of the Double Laser Emission Occurring in 1.55- $\mu \mathrm{m}$ InAs-InP (113)B Quantum-Dot Lasers," IEEE Journal of Quantum Electron., 43(9), 810-816 (2007). 
[19] Grillot, F., Veselinov, K., Gioannini, M., Montrosset, I., Even, J., Piron, R., Homeyer, E., and Loualiche, S., "Spectral analysis of $1.55 \mu \mathrm{m}$ InAs-InP(113)B quantum-dot lasers based on a multipopulation rate equations model," IEEE Journal of Quantum Electron., 45(7), 872-878 (2009).

[20] Miska, P. , Even, J., Dehaese, O., and Marie, X., "Carrier relaxation dynamics in InAs/InP quantum dots," Appl. Phys. Lett. 92, 191103 (2008).

[21] Bockelmann, U., and Egeler, T., "Electron relaxation in quantum dots by means of Auger processes," Phys. Rev. B, Condens. Phys. 46, 15574-15577 (1992).

[22] Agrawal, G. P., and Dutta, N. K., [Long Wavelength Semiconductor Lasers] , Van Nostrand Reinhold, New York, 240-244 (1986).

[23] Sugawara, M., Hatori, N., Ishida, M., Ebe, H., and Arakawa, Y., "Recent progress in self-assembled quantum-dot optical devices for optical telecommunication: Temperature-insensitive $10 \mathrm{Gbs}^{-1}$ directly modulated lasers and $40 \mathrm{Gbs}$ ${ }^{1}$ signal-regenerative amplifiers, " J. Phys. D, Appl. Phys. 38 (13), 2126-2134 ( 2005).

[24] Bhattacharya, P., and Mi, Z., "Quantum-dot optoelectronic devices," Proc. IEEE 95 (9),1723-1740 (2007)

[25] Krebs, R., Klopf, F., Rennon, S., Reithmaier, J.P., and Forchel, A., "High frequency characteristics of InAs/ GalnAs quantum dot distributed feedback lasers emitting at $1.3 \mu \mathrm{m}$," Electron. Lett. 37 (20), 1223-1225 (2001)

[26] Massé, N. F., Homeyer, E., Adams, A. R., Sweeney, S. J., Dehaese, O., Piron, R., Grillot F., Loualiche, S., "Temperature and pressure dependence of the recombination processes in 1.5 $\mu \mathrm{m}$ InAs/InP (311)B quantum dot lasers, " Appl. Phys. Lett. 91, 131113-131115 (2007).

[27] Su, C. B., Eom, J., William, C., Rideout, C., Lange, H., Kim, C. B., Lauer, R. B., and Lacourse, J. S., "Characterization of the Dvnamics of Semiconductor Lasers using Optical Modulation, " IEEE Journal of Quantum Electron., 28(1), 118-127 (1992). 